\title{
FLEXOR AND EXTENSOR TENDON INJURIES
}

\author{
L. K. PRETORIUS, M.B., B.A.O., B.Ch., F.R.C.S. (Ireland)*
}

This discussion of flexor and extensor tendon injuries of the hand has been adapted to serve as a practical guide for physiotherapists.

\section{TYPE CLASSIFICATION}

Injuries are classified as 1. Tidy wounds, 2. Untidy wounds. 3. Time factor: 3.1 early; 3.2 late. 4. Other structures damaged.

Type 1: $A$ tidy wound is a clean incised wound with no laceration or contusion. The object was either a sharp knife or the equivalent of a glass shard.

Type 2: An untidy wound is a more ragged type of injury, where the skin and deep structures are cut in a ragged fashion and contusion is present.

Type 3: Time Factor: 3.1 Early presentation, i.e. within $6-12$ hours after injury: there is a strong indication for thorough debridement and primary suture, especially if this wound is of Type 1 and has been cleanly bandaged. 3.2 Late presentation: no matter what the type the wound should be debrided and then packed with an antibiotic pack. The wound should be re-inspected after 48 hours. A decision may then be made about a further procedure, either primary suture, delayed suture, or no suture until healed.

Type 4: Other structures damaged: If possible all structures such as nerves and tendons should be repaired at the same sitting.

\section{BASIC PRINCIPLES OF MANAGEMENT}

1. Decontaminate the wound and surrounding skin, i.e. convert the untidy wound into a tidy wound. 2 . Excise all contused tissue, including any non-viable or doubtful tissue. 3. Delay repair if necessary for a period of up to 10 days. Repeated inspections in theatre and debridement should be done if necessary and the wounds must be packed with damp antibiotic, i.e. impregnated swabs, 4. Repair all structures at one sitting. 5 . Do not regard antibiotics as a substitute for a thorough debridement. This is axiomatic.

Choice of approach depends on the expertise of the surgeon and on the facilities available.

Primary repair is the method of choice. A tidy type of wound of less than 12 hours standing, may be dealt with by an immediately primary repair with thorough debridement. The wound must be sufficiently enlarged to allow good access and all structures should be repaired at one sitting. A careful no-touch technique is used.

Debridement and pack and delayed primary suture. If the surgeon is not able or willing to repair the structures, he may decide to pack the clean wound with impregnated antibiotic swabs and transfer the patient to the nearest hospital or even a distant specialist hospital, provided that the delay is not too long. The wound is then re-examined on arrival at the definitive hospital and is either repacked or a delayed primary suture is performed. This delay may safely be up to 10 days.

Skin closure afier debridement. No attempt is made to suture deep structures. When wound healing has occurred, secondary suture is performed from three to six weeks afterwards. This used to be the

* Senior Lecturer, Chief Orthopaedic Surgeon, Department of Orthopaedics, University of Stellenbosch, Tygerberg and Lady Michaelis Hospitals. standard procedure but is no longer accepted as ideal treatment unless dictated by circumstances such as poor facilities, poor general health of the patient, a non-expert surgeon.

Skin closure and grafing several months later. Once again, this used to be the standard procedure in the days when primary suture was deemed dangerous because of the possibility of infection. Unfortunately many cases are still seen in which perforce this is all that can be done.

Various newer methods of improving the gliding capability of the grafted tendon have been developed. Amongst these is silicone rod implantation which creates a new tunnel for the graft. The graft survives not only by being placed in tissue fluid in its new position, but also by the ingrowth of nev blood vessels and so-called adhesions which form and bind the graft to its bed. Often in these cases a tenolysis is necessary to obtain movement, and more often than not the result is less than satisfactory.

\section{Methods of Suture in Use:}

1. Holding-type suture (advocated by Kessler). (Fig. 1.) This suture is strong and reliable. 2. Holding-type suture (advocated by Bunnell) (Fig. 2.). This suture is strong and reliable. 3. Holding-type suture with buried knot (advocated by Kleinert) (Fig. 3). This suture is weak and needs to be well protected to achieve success. A circumferential 6-zero prolene suture is used to tidy up the suture line. 4. Intratendinous removable type suture (as used in Tygerberg Hospital) (Fig. 4). A circumferential 6-zero prolene suture is used to tidy up the suture.

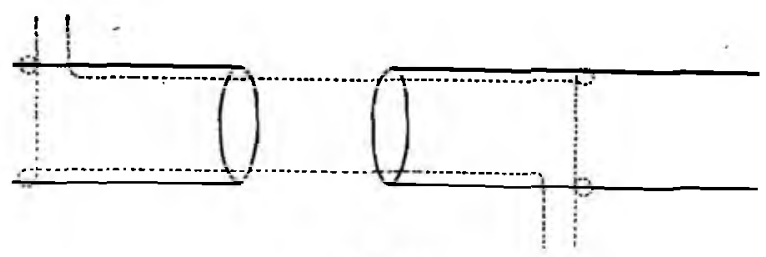

Fig. 1.

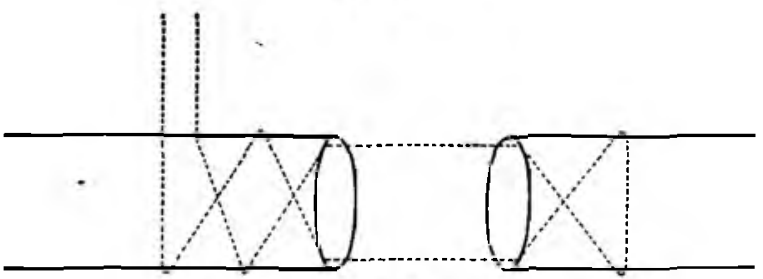

Fig. 2.

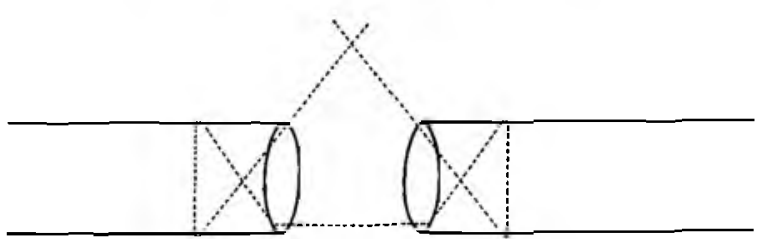

Fig. 3. 

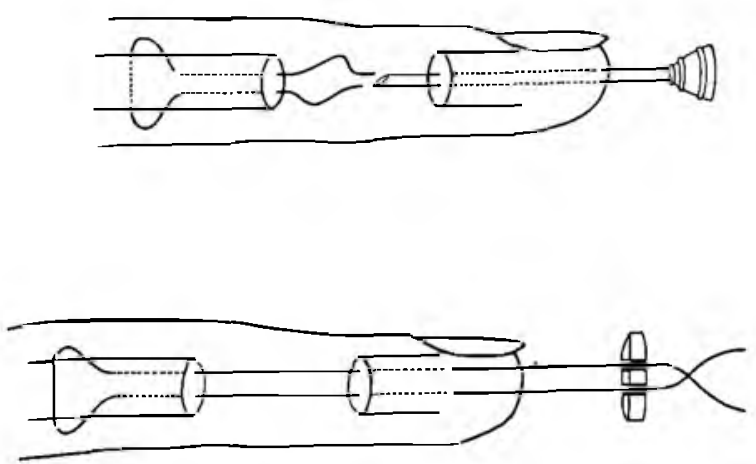

Fig. 4.

\section{SPECIFIC AREAS OF FLEXOR TENDON REPAIR}

Zone I. The area where a deep cut involves the exor digitorum profundus (F.D.P.) tendon only and here is no damage to the flexor digitorum sublimis (F.D.S.).

If the distal stump is less than $1,5 \mathrm{~cm}$ in length, it may be shortened or excised, and the proximal stump advanced and re-implanted into the distal phalanx. This is done to avoid the presence of a suture line in the pulley system. The re-implantation must be firm and is usually made into bone. A pull-out type of suture over an external button is used.

If the distal stump is longer than $1,5 \mathrm{~cm}$ then excision is not done or advised, for this advancement would result in a severe flexion deformity of the finger. A decision must now be taken as to whether to suture the tendon or to do a tenodesis of the distal interphalangeal joint. Under no circumstances should one interfere with the action of the normal F.D.S.

If the F.D.P. tendon has retracted into the palm, an appropriate incision will locate it and it may be rethreaded through the pulley system to be re-implanted, provided that F.D.S. is not compromised. A F.D.P. that has retracted into the palm and has lain there for longer than four weeks should not be used but should be excised. In this case the intratendinous vasculature has been compromised and the tendon is likely to rupture on use owing to patchy necrosis; the exception may be in the young child in whom such a endon is often in an extremely good condition and $\checkmark$ ay be used.

Zone II. "No man's land" of Bunnell, or "some man's land" of Kleinert and others.

Of the various techniques available, Kleinert's technique is the most attractive. Kleinert does a primary suture and then either excises a window in the pulley system where it lies over the suture line or else carefully repairs the damage to the pulley sheath. The hand is then placed in slight flexion at the wrist and a backcast is applied to prevent metacarpo-phalangeal extension of more than $45^{\circ}$ from full flexion. Traction elastics are attached to the fingernails of the affected fingers and tied to an anchorage at the forearm

Exercise may then begin immediately post-operatively, active extension only being used. This synergistically relaxes the flexors, allowing them to be moved passively more distally. When the extensors are relaxed the elastics will flex the fingers and thus no active flexion occurs with undue strain on the suture line.

All this prevents gross adhesion formation at the suture line, i.e. between the suture line and the flexor sheath and so facilitates eventual movement without the need for a tenolysis, although the latter may sometimes be necessary.
In Zone II either the F.D.S. and the F.D.P. are repaired or the F.D.S. is excised.

The post-operative period is carefully checked and controlled by the surgeon and the physiotherapist for four to six weeks post-operatively, whereupon the splint is removed and active flexion permitted.

Zone III. This is the area of the palm proximal to the pulley system and distal to the deep flexor retinaculum of the wrist. Here both F.D.S. and F.D.P. are repaired by a Kessler or a Bunnell suture and if necessary the lumbrical muscle may be used to conceal the suture line in the F.D.P. This is a good procedure resulting in less adhesion formation, but may result in the "lumbrical positive" finger. This phenomenon occurs through fibrosis or shortening of the lumbrical muscle; when finger flexion is attempted, simultaneous finger extension occurs because the pull on the flexor is transmitted via the lumbrical and the lateral band to the extensor apparatus.

If the lumbrical is used as a "wrap around", then its tendon should be sectioned to prevent the development of this "lumbrical positive" finger. The Kleinert-type of post-operative regime is followed (elastics and cast).

Zone IV. Carpal tunnel area. There is a concentration of nine tendons and one nerve into an area where the tip of the normal middle finger will just fit tightly. If the F.D.S. and the F.D.P. are both severed, the procedure is to suture F.D.P. only and excise the F.D.S.

Alternatively one may decide to suture the proximal F.D.S. motor system to the distal F.D.P. tendon system in an attempt to obtain more individualised finger action. The choice depends on the surgeon and the technical feasibility. If possible a portion of the flexor retinaculum should be left as a pulley. Kessler or Bunnell-type of sutures are used for these cases.

Zone $V$. The forearm. In this zone all structures should be repaired. Kessler or Bunnell-type sutures are indicated.

\section{EXTENSOR TENDON SUTURE}

The same general principles apply as for flexor tendon injuries. Because of the flat shape of the tendon and its more delicate nature, a Kessler or Bunnell-type of suture is preferable. Post-operatively the hand is placed in a full relaxed position in a plaster of Paris cast for four to six weeks. If a pulley or retinaculum is involved, it should be excised over the excursion of the suture line.

The index and the little fingers each have an extra extensor tendon in addition to the extensor digitorum communis (E.D.C.). These are very useful clinically to substitute for irreparable extensor tendons and are otfen used as transfers. Although there is no digital pulley system on the extensor surface of the finger comparable with the flexor system, the anatomical arrangement is quite complicated.

Injuries involving the implantation of the extensor tendon into the distal phalanx create a lesion known as "mallet finger". This is a typical flexion deformity of the terminal joint due to the unopposed action of the F.D.P. The extensor tendon may be avulsed or incised, or it may be undamaged, in which case the deformity is due to a fracture in the epiphyseal line of the distal phalanx. This last injury occurs in children and all mallet fingers must undergo X-ray examination in order to differentiate this type of presentation.

Repair is effected by re-implanting the tendon apparatus into the terminal phalanx by direct suture or by replacing the avulsed bone accurately. The position is then maintained by transfixing the joint with a Kirschner wire for six weeks. Mobilization is effected after removal of the wire, but is very slow and 
often incomplete.

The traumatic Boutonnière lesion: In this case the injury is to the middle slip of the extensor tendon inserted into the middle phalanx on its dorsal aspect. When this occurs the triangular ligament uniting two lateral tendons prior to their fusion is also disrupted and the proximal interphalangeal joint (P.I.P.), because of the absence of an extensor, progresses into flexion. This process is slow and due to gradual volar displacement of the lateral bands over the area of the P.I.P. joint until they now act as flexors rather than extensors of this joint. As the P.I.P. joint proceeds into flexion, so the distal interphalangeal (D.I.P.) joint assumes a position of extension as a result of the increased pull of the displaced lateral bands on their eventual insertion into the terminal phalanx. The lesion may be grouped into mobile and fixed versions. In the mobile version, the P.I.P. joint is still mobile and may be passively placed into full extension. In the fixed version, the P.I.P. joint is no longer able to be moved passively into extension.

1. Treatment of the mobile version: This injury should be treated aggressively whilst still in the mobile phase, and either efficiently splinted, or surgically corrected and splinted. Splintage must be maintained for six weeks.

An efficient splint is one which holds the P.I.P. joint in extension, whilst allowing flexion and extension at the D.I.P. joint. This action tends to close the gap in the triangular ligament and to promote healing and may be used as a conservative method of treatment.

In the author's opinion, surgical correction is effective and a more satisfactory result is obtained in that the correction is anatomical. The P.I.P. joint is transfixed with a Kirschner wire which ensures adequate healine After six weeks the wire is removed and active mobill. zation is commenced. It often takes up to two months for the finger to be completely mobilized.

2. Treatment of the fixed version: The fixed type is very difficult to treat and attempts must be made to mobilize the finger before operation. Various forms of dynamic finger splints are used and all are effective if correctly designed and correctly applied. No surgery should be attempted until correction is obtained. If correction has to be obtained by surgical means, the end result is often extremely disappointing and the prognosis of arthrodesis of the P.I.P. joint is a distinct possibility.

\section{Bibliography:}

Weeks, P. M., Christie Wray, R., (1973). Management Acute Hand Injuries, C. V. Mosby Company, S Louis.

Rank, B. K., Wakefield, A. R., Hueston, J. T., (1973). Surgery of Repair as Applied to Hand Injuries, 4th Ed., Churchill Livingstone, Edinburgh and London. Boyes, $\mathbf{J}$. H., personal communication.

Kleinert, H. E., personal communication. 